\title{
Preparation of Single- and Few-Layer Graphene Sheets Using Co Deposition on SiC Substrate
}

\author{
Cun Li, Dandan Li, Jingjing Yang, Xiaopeng Zeng, and Wenxia Yuan \\ Department of Chemistry, School of Applied Science, University of Science and Technology Beijing, Beijing 100083, China \\ Correspondence should be addressed to Wenxia Yuan, wxyuan@ustb.edu.cn
}

Received 29 January 2011; Revised 16 May 2011; Accepted 22 May 2011

Academic Editor: Zhi Li Xiao

Copyright () 2011 Cun Li et al. This is an open access article distributed under the Creative Commons Attribution License, which permits unrestricted use, distribution, and reproduction in any medium, provided the original work is properly cited.

Single- and few-layer graphene sheets were fabricated by selective chemical reactions between Co film and SiC substrate. A rapid cooling process was employed. The number of layers and crystallinity of graphene sheets were controlled by process parameters. The formation mechanism of graphene was highly sensitive to carbon diffusion. Free carbon precipitated and then moved across the product layer that was composed mainly of cobalt-silicides. The graphene layer formed homogeneously on the surface and then transferred to the other substrate. This could provide a method for high-quality fabrication of wafer-sized graphene sheets.

\section{Introduction}

A highly crystalline material, graphene is represented as a two-dimensional (2D) building block of carbon allotropes with honeycomb lattice [1]. Since its discovery in 2004, graphene has drawn intense experimental and theoretical interest, thanks to its very unique properties [2-4].

Currently, graphene sheets are prepared by different methods such as mechanical cleavage of graphite using adhesive tapes [2], chemical reduction of graphite oxide $[5,6]$, epitaxial growth (EG) of graphene by thermal graphitization of $\mathrm{SiC}$ [7], chemical vapor deposition (CVD) of hydrocarbon gases on transition metals [8], exfoliation of graphite by sonication [9], quenching [10], and wet ball milling [11]. Of these methods, EG grown on $\mathrm{SiC}$ has attracted much interest owing to its potential as a viable candidate for post CMOS electronics. Recently, an alternative method using SiC as carbon source for graphene sheet preparation has been demonstrated $[12,13]$. In these works, Ni film deposited on $\mathrm{SiC}$ single-crystal substrate [12] or $\mathrm{SiC}$ amorphous thin film on $\mathrm{SiO}_{2}$ substrate [13] was used as a catalyst for $\mathrm{SiC}$ graphitization. Graphene sheets were synthesized by rapid thermal annealing and were later exfoliated by acid etching to remove the Ni silicide layer. The formation of graphene is based on the reaction mechanism that $\mathrm{C}$ releases owing to $\mathrm{Ni}$ silicide formation reaction forms a thin graphite layer on the top of the surface [14]. More recent work further studied the influence of $\mathrm{Ni}$ thickness for the quality of graphene on $\mathrm{SiC}$ substrate $[15,16]$. This method widens the vision for graphene preparation using both $\mathrm{SiC}$ and transition metals, mainly through the CVD method. However, detailed understanding of growth kinetics and production control is highly desired to accommodate large-scale quality graphene.

This paper presents a route for graphene preparation by using $\mathrm{SiC}$ substrate reaction with Co film instead of Ni. Co can also act as a catalyst for graphitization of SiC. However $\mathrm{SiC}$ decomposes at higher temperature by catalyzed by Co, which could lower defects in formation of graphenes, compared with that by Ni. Additionally, unlike in fast heating (cf. [12, 13]), fast cooling following annealing is used to suppress the surplus precipitated $\mathrm{C}$ on top of the produced silicon-cobalt layer. The formation mechanism of graphene on $\mathrm{Co} / \mathrm{SiC}$ contacts is revealed by the morphology and structure analysis of the interface. The graphene layer can form on the surface, providing potential methods for the fabrication of wafer-sized graphenes. Instead of dredging up graphene layers after acid etching [12, 13], supportive coating of a poly(methyl methacrylate) (PMMA) film is used on graphene [17] in order to enhance transfer efficiency to the other substrate.

\section{Experimental}

2.1. Preparation Procedure. Commercial grade n-type $6 \mathrm{H}$ $\mathrm{SiC}$ single-crystal wafers (surface roughness $<0.5 \mathrm{~nm}$, TankeBlue Semconductor Company) were used as substrates. Co 


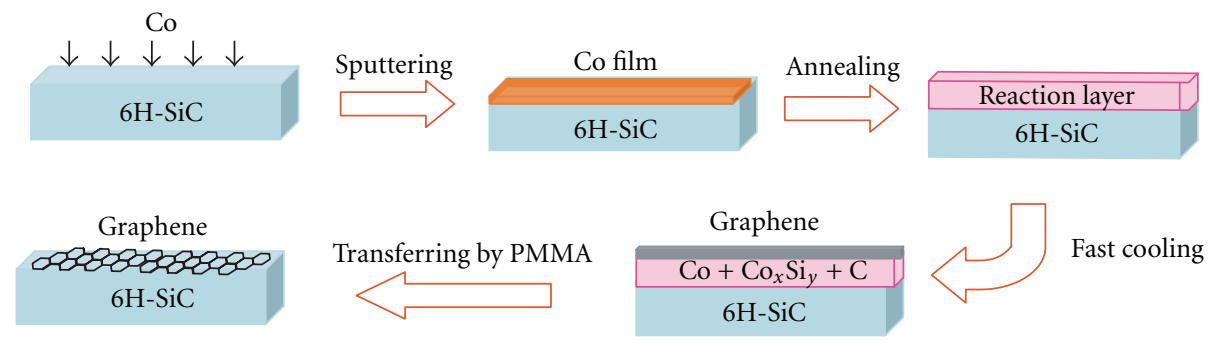

FIGURE 1: Schematic diagram of graphene formation process.

layers (200-300 nm thick, 99.99 wt.\% pure) were deposited by AJA ATC-1800F magnetron sputtering on a polished SiC silicon-terminated surface. Then, samples were subjected to thermal annealing in a vacuum greater than $5 \times 10^{-5} \mathrm{~Pa}$ or in ambient pressure under an Ar environment. Sample annealing temperatures were within $900-1000^{\circ} \mathrm{C}$. Water bath was used to cool quickly the annealed samples to room temperature.

Graphene on the surface was transferred to the other substrate. The transferring process used PMMA film as support. Prior transferring, a layer of PMMA (MicroChem, 950,000 MW, 9-6wt.\% in anisole) was spin-coated on the sample surface. Afterwards, detachment of the PMMA/graphene layer from the initial surface was done by etching partially the $\mathrm{Co} /$ silicides layer with a $\mathrm{HNO}_{3}$ (14 wt.\%) solution for more than $24 \mathrm{~h}$. Upon release, the substrate was placed in distilled water at room temperature where manual peeling completely detached the PMMA/graphene membrane from the substrate. Finally, the PMMA membrane was dissolved with acetone. Graphene settled on another SiC substrate. After the transfer, the original SiC pieces were polished for subsequent use.

The whole preparation process for graphene sheets is illustrated in Figure 1.

2.2. Characterization. X-ray diffraction (XRD) method was used to identify the phase formation of the $\mathrm{Co} / \mathrm{SiC}$ contact by a Philips X'PERT Pro X-ray ploycrystal diffractometer, where $\mathrm{Cu} \mathrm{K} \alpha$ radiation was employed. Raman microspectroscopy (Nicolet), nano-scanning Auger electron spectroscopy (AES, ULVAC PHI-700), atomic force microscopy (AFM, Dimension $\mathrm{V}$ ), and field-emission scanning electron microscopy (SEM, SUPRA 55) were used to characterize the graphene and morphology of the contacts. The electrical property of deposition contact was carried out with Keithley 220 Current Sources Power Supplies and Keithley 2000 Voltage Sources Power Supplies.

\section{Results and Discussion}

Identification of the interfacial reaction products obtained after each annealing was performed by reference to powder diffraction files. The XRD pattern is given in Figure 2 for the sample annealed at $900^{\circ} \mathrm{C}$ for $10 \mathrm{~min}$. It indicates that

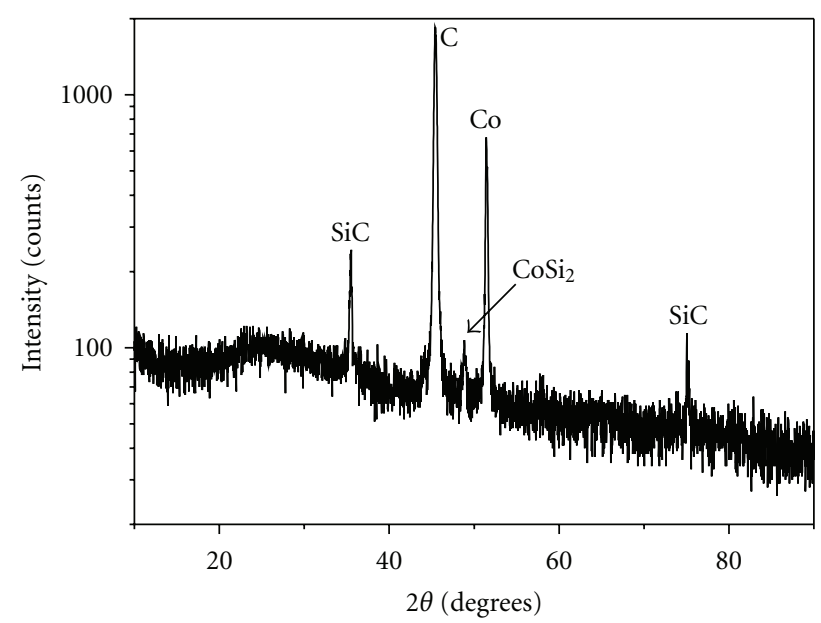

FIGURE 2: XRD patterns of the $\mathrm{Co} / \mathrm{SiC}$ sample annealed under $900^{\circ} \mathrm{C}$ for $10 \mathrm{~min}$.

anneals process leads to the formation of Co silicides, for example, $\mathrm{CoSi}_{2}$, and free carbon. Referring to the Gibbs free energy of formation of $\mathrm{Co}$ silicides and $\mathrm{SiC}$, the interface reaction ( $\mathrm{Co}+\mathrm{SiC} \rightarrow \mathrm{Co}_{x} \mathrm{Si}_{y}+\mathrm{C}$ ) is thermodynamically favored, since Co silicides have negative Gibbs free energies than that of $\mathrm{SiC}$ [18]. Figures 3(a) and 3(b) give SEM images of the sample. Combined with the SEM image of the cross-section of a product layer (Figure 3(b)), the grains became clearly visible. The surface of the layer is relatively flat, at which a contact layer is thicker than the as-deposited $(200 \mathrm{~nm})$. The EDS analysis of the top surface in the reaction region detected the high carbon contents (about 58\%) of the layer. It is deduced that the graphene layer formed on the top surface and the unreacted cobalt stayed with the produced cobalt silicides in the production layer. Raman scattering patterns (532 $\mathrm{nm}$ excitation) from light contrast positions in the sample (Figure 3(a)) is reshown as Figure 3(c). The 2D band peak fittings with single Lorentzian curve centered at $2680 \mathrm{~cm}^{-1}$ is presented in Figure 3(d).

It is well known that the most striking differences of Raman features in bulk graphite and graphene sheets are as follows: (1) $2 \mathrm{D}$ intensity over $\mathrm{G}$ intensity ratio $\left(I_{2 \mathrm{D}} / I_{\mathrm{G}}\right) \geq 1$, and $2 \mathrm{D}$ full width at half maximum (FWHM) at $\sim 30 \mathrm{~cm}^{-1}$ for monolayer graphene; (2) $2 \mathrm{D}$ peak at $\sim 2700 \mathrm{~cm}^{-1}$, which is single and sharp for single-layer graphene; (3) G peak 


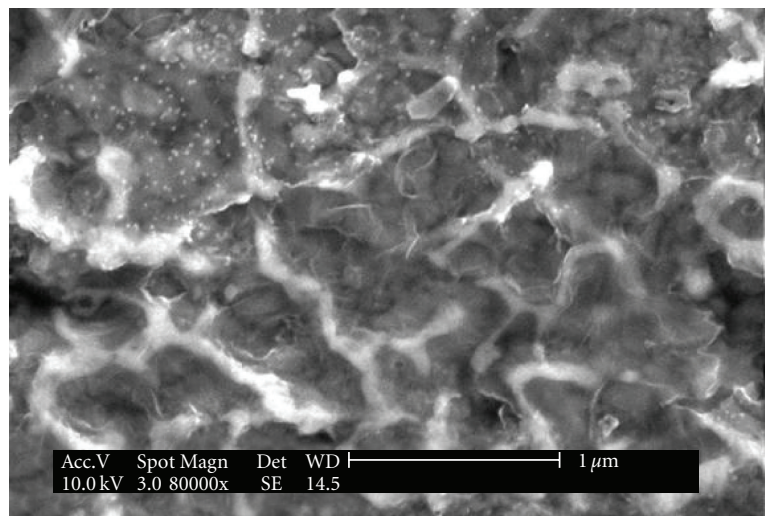

(a)

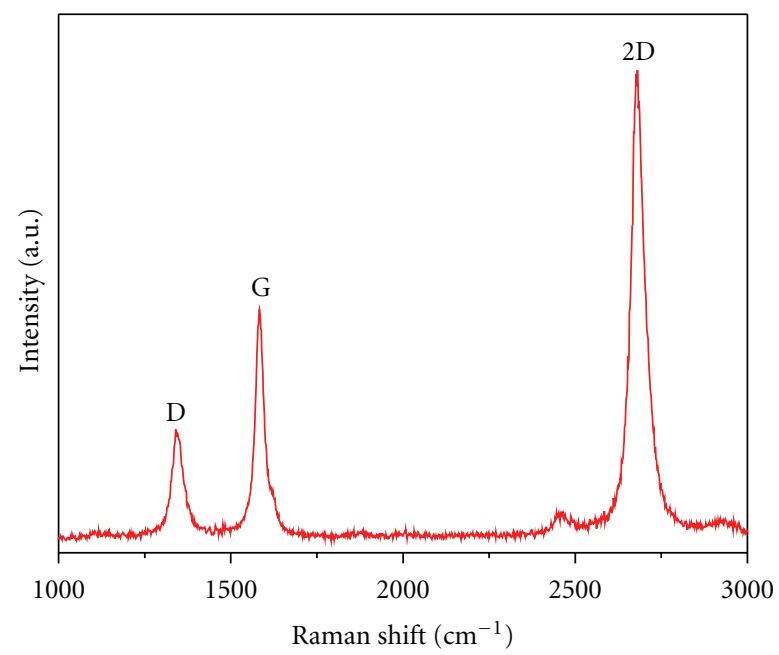

(c)

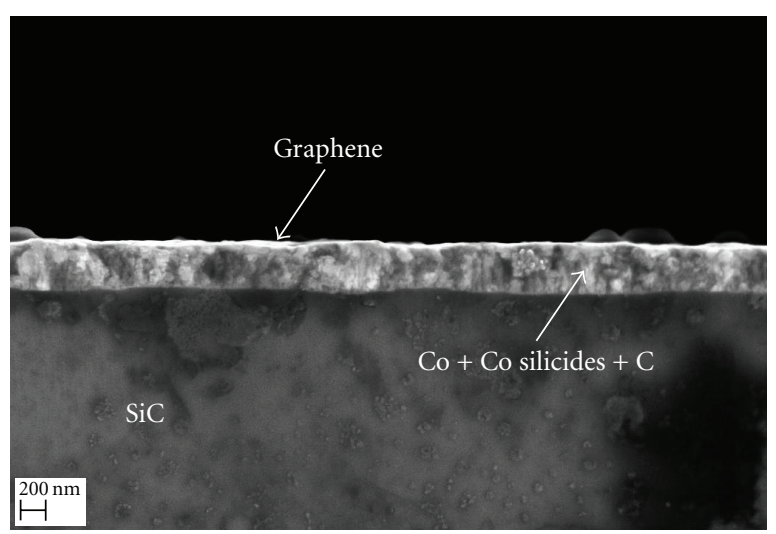

(b)

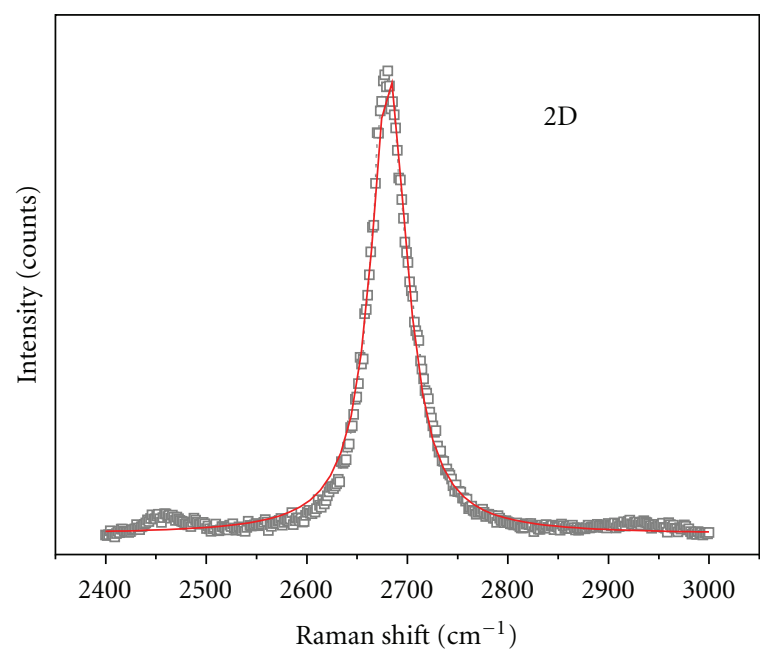

(d)

Figure 3: SEM images of (a) surface morphology of produced layers. (b) The cross-section of the sample. (c) The corresponding Raman spectra $\left(532 \mathrm{~nm}\right.$ excitation) recorded from light contrast region. Intensity ratio of $I_{2 \mathrm{D}} / I_{\mathrm{G}}=2$. (d) $2 \mathrm{D}$-band peak fittings with a single lorentzian curve centered at $2680 \mathrm{~cm}^{-1}$.

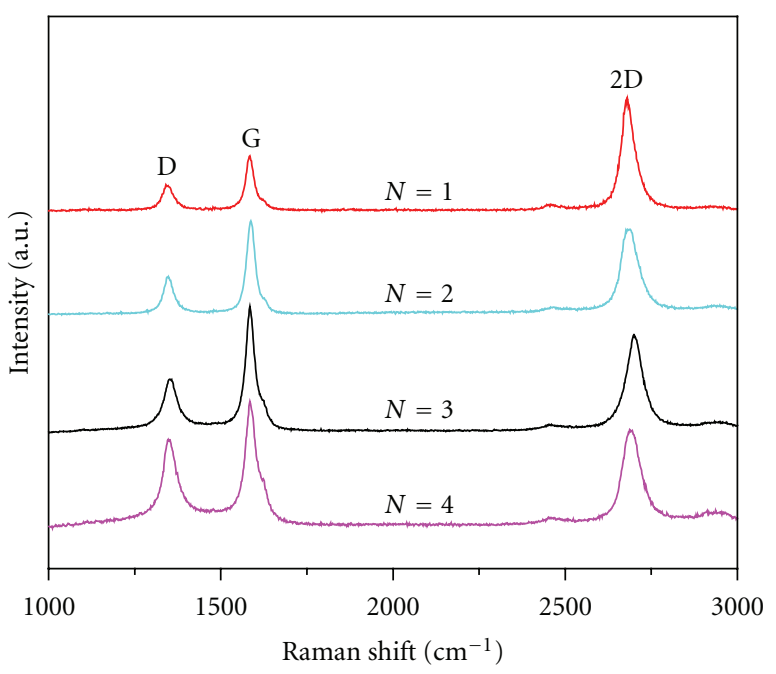

Figure 4: Evolution of D, G, and 2D peak of our samples as a function of number of graphite layers for $532 \mathrm{~nm}$ excitations. position of graphene with lower wave number than graphite. Our sample showed a clear D band at $\sim 1340 \mathrm{~cm}^{-1}$; G band at $1580 \mathrm{~cm}^{-1} ; 2 \mathrm{D}$ band at $2680 \mathrm{~cm}^{-1}$. The FWHM of the $2 \mathrm{D}$ peak was $30 \mathrm{~cm}^{-1}$, a value larger than that for the exfoliated monolayer graphene [19]. The D band is associated with defects. This could be attributed to the stress induced by the high-temperature process, which did not relax upon cooling. This phenomenon was also observed for graphene epitaxially grown on $\mathrm{SiC}$ [20]. Raman spectra in Figures 3(c) and $3(\mathrm{~d})$ indicate that this region of the sample comprised a monolayer graphene. The shape of the $2 \mathrm{D}$ peak and $I_{2 \mathrm{D}} / I_{\mathrm{G}}$ allowed this study to evaluate the number of graphene layers [21]. Raman scattering of the samples showed that 2D peaks became wider and lower in intensity with the number of layers (Figure 4). The FWHM of the 2D band was estimated to be $\sim 60 \mathrm{~cm}^{-1}$ for a four-layer graphene sheet sample [21]. This reveals that thin graphene regions (1-4 layers) were present in the light-colored areas, and these contrasted with the thicker graphitic regions (see dark areas in Figure 3(a)). 


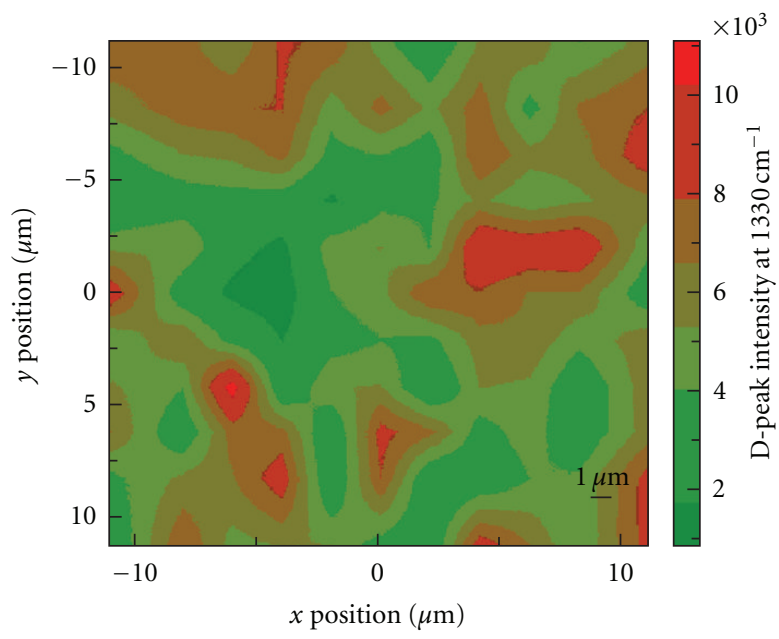

(a)

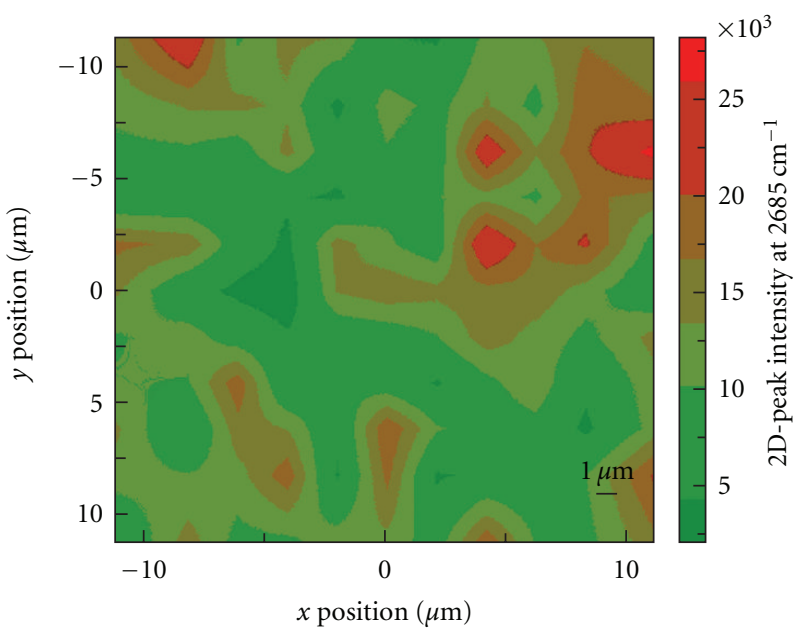

(c)

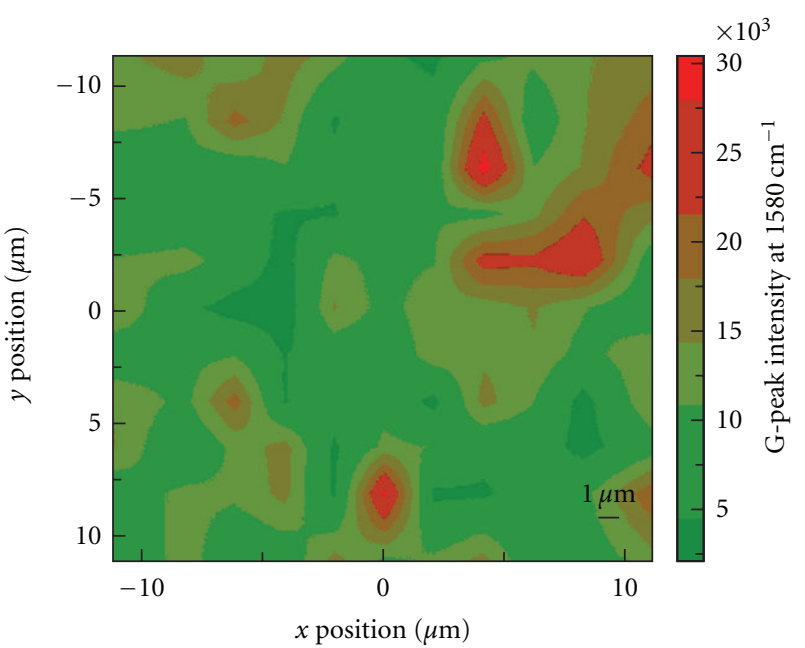

(b)

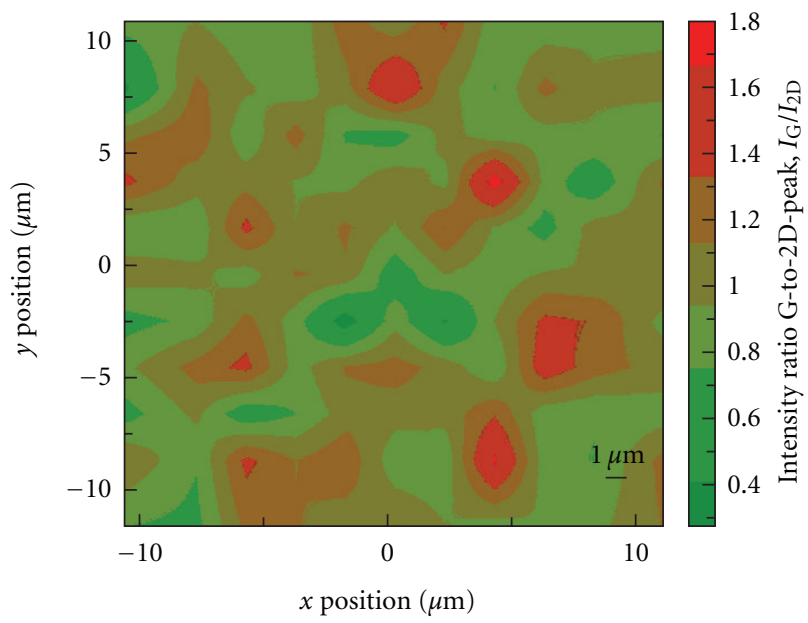

(d)

Figure 5: Raman mapping data for an area of $20 \times 20 \mu \mathrm{m}^{2}$ : (a) D-peak $\left(1350 \mathrm{~cm}^{-1}\right)$ intensity, (b) G-peak $\left(1600 \mathrm{~cm}^{-1}\right)$ intensity, (c) $2 \mathrm{D}$-peak $\left(2700 \mathrm{~cm}^{-1}\right)$ intensity, (d) Intensity ratio of the G-peak to the $2 \mathrm{D}$-peak.

Raman mapping was performed to obtain further information on film uniformity. Figure 5 shows the corresponding Raman intensities of (a) D peak at around $1350 \mathrm{~cm}^{-1}$, (b) $G$ peak around $1580 \mathrm{~cm}^{-1}$, and (c) $2 \mathrm{D}$ peak at $2700 \mathrm{~cm}^{-1}$ for an area of $20 \times 20 \mu \mathrm{m}^{2}$. The intensity ratio of $\mathrm{G}$ peak to $2 \mathrm{D}$ peak is shown in Figure 5(d). With single-layer areas evident, some areas of the surface are few-layer graphene while others are graphite.

The topography imaging of transferred graphene sheet was performed by AFM measurement as shown in Figure 6 . It shows that the average thickness of graphene is $3 \mathrm{~nm}$. Inz Figure 6(b), the wrinkles are elevated. It can be attributed to folding of the graphite induced by the different expansion coefficients of metal and graphite during cooling down after the high-temperature process [13]. The Raman spectra of transferred graphene were observed (Figure 6(c)), showing that few-layer graphene was exfoliated successfully onto the $\mathrm{SiC}$ substrate. The AFM image in Figure 6(a) shows that the edge of transferred graphene film did not fit well with
$\mathrm{SiC}$ substrate. The previous studies showed that graphene is combined with $\mathrm{SiC}$ through buffer layers. These buffer layers are defect-rich $\mathrm{SiC}$ layers. The interface between graphene and buffer layers is characterized by existence of breaking bonds compared over SiC layer.

The current-voltage (I-V) characteristics of $\mathrm{Co} / \mathrm{SiC}$ contact annealed at $1000^{\circ} \mathrm{C}$ for $1 \mathrm{~min}$ and transferred graphene film on $\mathrm{SiC}$ substrate were performed by four-probe method as shown in Figure 7. Postdeposition annealing at $1000^{\circ} \mathrm{C}$ results in a sharp enhancement in the I-V curve compared to the as-deposited contact. The formation of graphene and Co silicides improves the I-V characteristics of contact [22]. The I-V curve of exfoliated graphene sheet on $\mathrm{SiC}$ substrat also shows a linear feature (Figure 7(b)), with a slope of three-order lower than that of $\mathrm{Co} / \mathrm{SiC}$ after annealed at $1000^{\circ} \mathrm{C}$. The reason might come from the inhomogeniety of transferred graphene film on $\mathrm{SiC}$ substrate (see Figures 6(a) and 6(b)).

To understand better the formation mechanism of graphene sheets using $\mathrm{Co}$ as catalyst for $\mathrm{SiC}$ decomposition, 

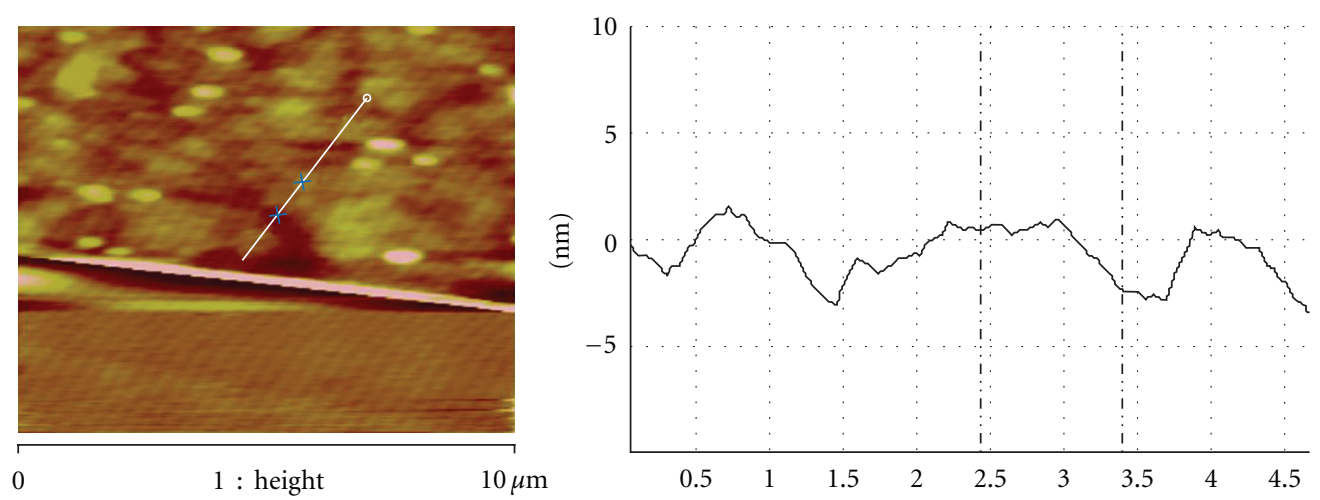

(a)

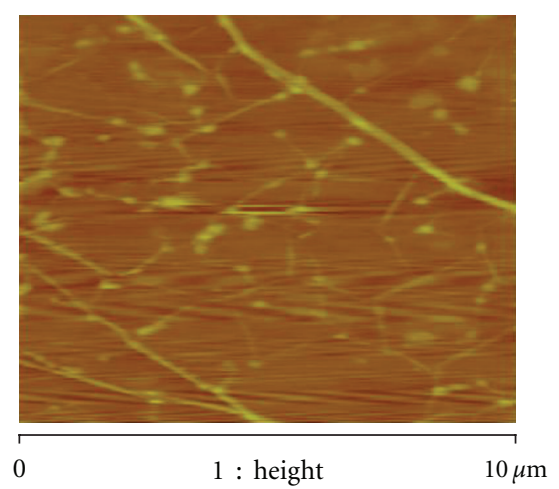

(b)

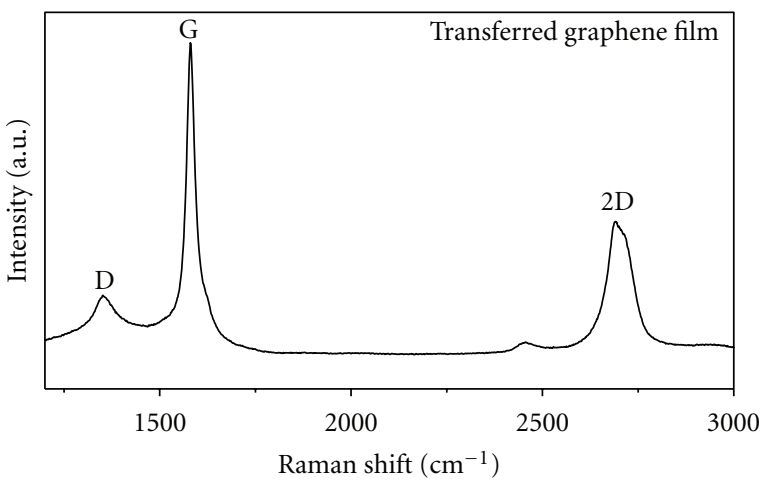

(c)

FIGURE 6: AFM images of the transferred graphene sheet on SiC substrate: (a) interface edge of graphene contacting with SiC, (b) surface morphology of graphene film. The size of the selected area is $10 \times 10 \mu \mathrm{m}^{2}$, and (c) Raman spectra of the transferred graphene sheet on SiC substrate.

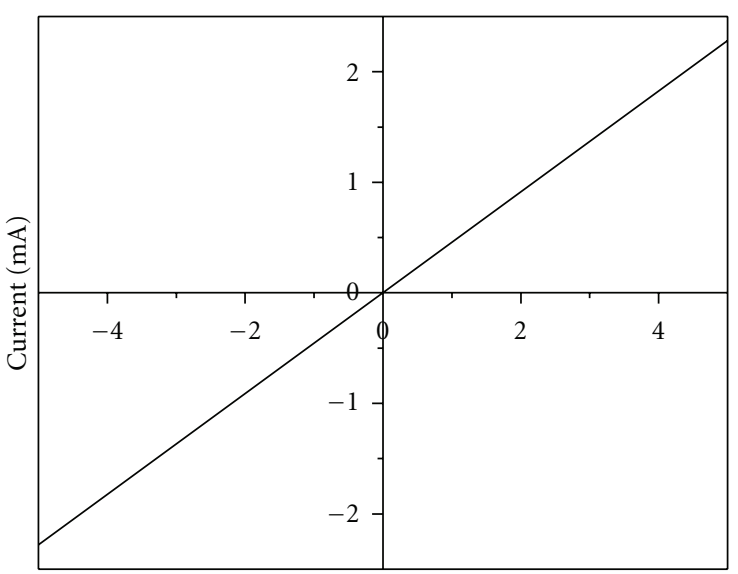

Voltage $(\mathrm{mV})$

(a) $\mathrm{Co} / \mathrm{SiC}$ sample annealed at $1000^{\circ} \mathrm{C}$

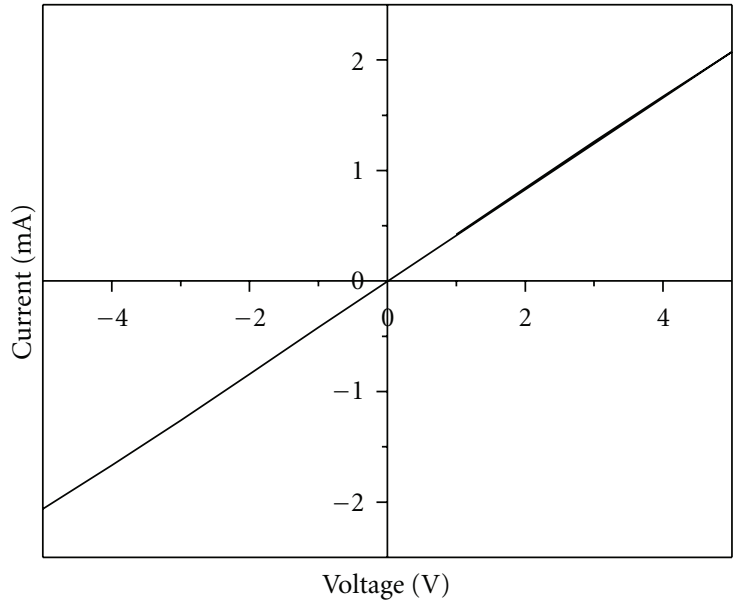

(b) Transferred graphene sheets on $\mathrm{SiC}$ substrate

Figure 7: Current-voltage characteristics of the samples: (a) $\mathrm{Co} / \mathrm{SiC}$ contact annealed at $1000^{\circ} \mathrm{C}$ for 1 min and (b) transferred graphene sheet on SiC substrate.

element distribution across the $\mathrm{Co} / 6 \mathrm{H}-\mathrm{SiC}$ contacts was measured using various annealing parameters. The AES depth of elemental distribution across the contact annealed at $1000^{\circ} \mathrm{C}$ for $10 \mathrm{~min}$ is shown in Figure 8 . Peaks were observable from the curves of $\mathrm{Co}$ and $\mathrm{Si}$ concentrations at the sputtering depth of $100 \mathrm{~nm}$, and these were about $53 \%$ and $22 \%$ of concentration, respectively. Meanwhile, C concentration decreased to minimum at the depth of $70 \mathrm{~nm}$ from the top surface with $80 \%$ content. Compared to asdeposited sample, it indicates that annealing process makes 


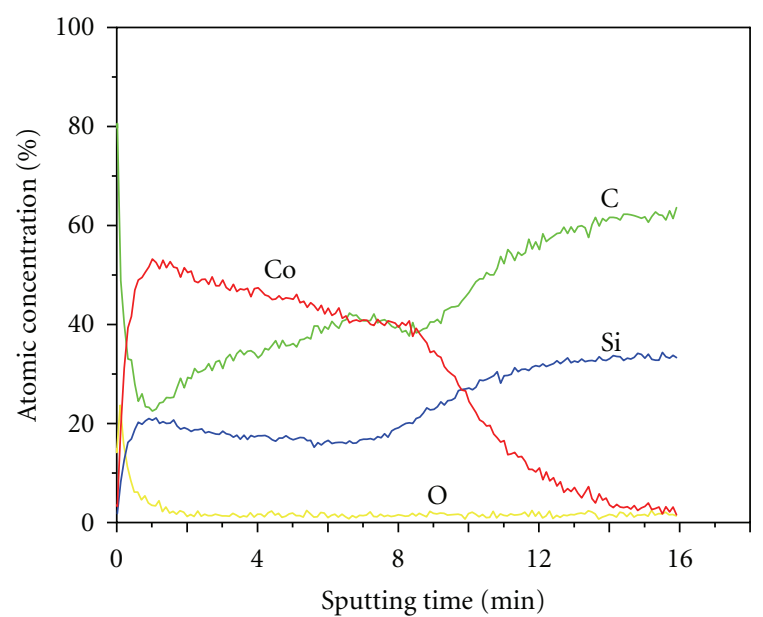

Figure 8: AES depth profile of the $\mathrm{Co} / \mathrm{SiC}$ contact annealed for 10 min under $1000^{\circ} \mathrm{C}$.

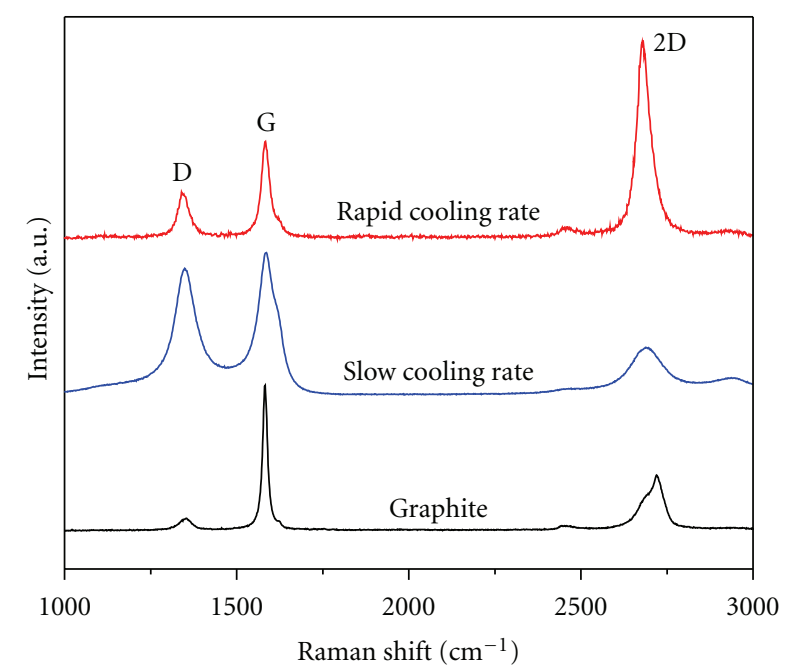

FIGURE 9: Raman spectra of segregated carbon with different cooling rates. Bulk graphite is used as a reference.

Co diffuse into the interface to react with $\mathrm{SiC}$ occurring at the interface. Co only reacts with $\mathrm{Si}$, whereas free $\mathrm{C}$ accumulates and finally diffuses throughout the loose product layer to the top surface due to low solubility in Co [10].

In a thick $(>100 \mathrm{~nm}) \mathrm{metal} / \mathrm{SiC}$ contact, the diffusion of species is the rate-determining step for the interfacial process [23]. Within the Co (200 nm thick)/SiC contact, the decrease of Co concentration from 86 to $14 \%$ was used to approximately determine thickness of diffusion layer according to the AES profiles. The results in Figure 8 show that the thickness of the diffusion layer (denoted as $x$ ) was $\sim 400 \mathrm{~nm}$. Diffusion-controlled kinetic features for the $\mathrm{Co} / \mathrm{SiC}$ contact followed the parabolic law wherein the square of $x$ is proportional to annealing time. When annealing duration was decreased, interface reaction was suppressed to a certain extent. Co was therefore not consumed completely; it also acted as a residual block layer for the carbon diffusion on the top surface.
Based on the proposed mechanism, the number of layers and graphene sheet crystallinity were tuned according to process parameters. Annealing duration and cooling rate are critical in carbon layer formation and efficient graphene layer separation from the substrate. With increasing reaction time, the 2D peaks of Raman spectra broadened, suggesting that thickness of the carbon layers diffused to the surface has become larger. A fast cooling step after annealing would decrease carbon diffusion rate and result in fewer amounts of carbon moving outward the reaction zone to form single- and few-layer carbons. The Raman spectra dependence on the cooling process after thermal annealing is illustrated in Figure 9. In a rapid cooling process, diffusion of surplus carbon from the surface was limited, resulting in the formation of monolayer graphene. Experiments also showed that the quality of graphene was not sensitive to vacuum and Ar atmosphere during annealing.

\section{Conclusions}

Single- and few-layer graphene sheets were successfully synthesized using a Co film that was selectively reacted with a $\mathrm{SiC}$ single-crystal substrate at $900-1000^{\circ} \mathrm{C}$, and assisted by rapid cooling. Graphene formation was based on controlling the reaction between $\mathrm{Co}$ and $\mathrm{Si}$ from the $\mathrm{SiC}$ substrate. The process produced carbon diffusion throughout the $\mathrm{Co} / \mathrm{Co}$ silicide layers. The formation mechanism reveals that optimal conditions for single- and few-layer graphenes resulted from tuning Co film thickness, annealing temperature/duration, and cooling rate. By using PMMA films, graphene could be transferred to the other substrate. The 1-4 layers of graphene, with a few defects or impurities, were obtained. Further optimization of the growth process should focus on higher uniformity of graphene sheets. The use of multilayer transition metals like $(\mathrm{Co}+\mathrm{Au})$ could provide better block layers for the available carbon, apart from ensuring better crystal orientation in the graphene sheets. It is expected to provide a simple and efficient strategy to prepare high-quality graphene with wafer size.

\section{Acknowledgments}

Financial support was provided by the National Natural Science Foundation of China (Grant No. 50772012 and 50972010) and the Fundamental Research Funds for Central Universities (FRF-TP-09-021B). Professor Liu Zhongfan from Peking University is grateful for the help of graphene transferring.

\section{References}

[1] A. K. Geim and K. S. Novoselov, "The rise of graphene," Nature Materials, vol. 6, no. 3, pp. 183-191, 2007.

[2] K. S. Novoselov, A. K. Geim, S. V. Morozov et al., "Electric field in atomically thin carbon films," Science, vol. 306, no. 5696, pp. 666-669, 2004.

[3] Y. Zhang, Y. W. Tan, H. L. Stormer, and P. Kim, "Experimental observation of the quantum Hall effect and Berry's phase in graphene," Nature, vol. 438, no. 7065, pp. 201-204, 2005. 
[4] A. H. Castro Neto, F. Guinea, N. M. R. Peres, K. S. Novoselov, and A. K. Geim, "The electronic properties of graphene," Reviews of Modern Physics, vol. 81, no. 1, pp. 109-162, 2009.

[5] F. Schedin, A. K. Geim, S. V. Morozov et al., "Detection of individual gas molecules adsorbed on graphene," Nature Materials, vol. 6, no. 9, pp. 652-655, 2007.

[6] F. Wang, Y. Zhang, C. Tian et al., "Gate-variable optical transitions in graphene," Science, vol. 320, no. 5873, pp. 206209, 2008.

[7] H. A. Becerril, J. Mao, Z. Liu, R. M. Stoltenberg, Z. Bao, and Y. Chen, "Evaluation of solution-processed reduced graphene oxide films as transparent conductors," ACS Nano, vol. 2, no. 3, pp. 463-470, 2008.

[8] S. Niyogi, E. Bekyarova, M. E. Itkis, J. L. McWilliams, M. A. Hamon, and R. C. Haddon, "Solution properties of graphite and graphene," Journal of the American Chemical Society, vol. 128, no. 24, pp. 7720-7721, 2006.

[9] Z. S. Wu, W. Ren, L. Gao, B. Liu, C. Jiang, and H. M. Cheng, "Synthesis of high-quality graphene with a pre-determined number of layers," Carbon, vol. 47, no. 2, pp. 493-499, 2009.

[10] J. Hass, W. A. De Heer, and E. H. Conrad, "The growth and morphology of epitaxial multilayer graphene," Journal of Physics Condensed Matter, vol. 20, no. 32, Article ID 323202, 2008.

[11] N. P. Guisinger, G. M. Rutter, J. N. Crain, P. N. First, and J. A. Stroscio, "Exposure of epitaxial graphene on $\mathrm{SiC}(0001)$ to atomic hydrogen," Nano Letters, vol. 9, no. 4, pp. 1462-1466, 2009.

[12] Z. Y. Juang, C. Y. Wu, C. W. Lo et al., "Synthesis of graphene on silicon carbide substrates at low temperature," Carbon, vol. 47, no. 8, pp. 2026-2031, 2009.

[13] J. Hofrichter, B. N. Szafranek, M. Otto et al., "Synthesis of graphene on silicon dioxide by a solid carbon source," Nano Letters, vol. 10, no. 1, pp. 36-42, 2010.

[14] T. Yoneda, M. Shibuya, K. Mitsuhara, A. Visikovskiy, Y. Hoshino, and Y. Kido, "Graphene on $\mathrm{SiC}(0001)$ and $\mathrm{SiC}(0001$ combining overline) surfaces grown via Ni-silicidation reactions," Surface Science, vol. 604, no. 17-18, pp. 1509$1515,2010$.

[15] A. A. Woodworth and C. D. Stinespring, "Surface chemistry of $\mathrm{Ni}$ induced graphite formation on the $6 \mathrm{H}-\mathrm{SiC}\left(\begin{array}{llll}0 & 0 & 0 & 1\end{array}\right)$ surface and its implications for graphene synthesis," Carbon, vol. 48, no. 7, pp. 1999-2003, 2010.

[16] Y. Cao, L. Nyborg, D. Q. Yi, and U. Jelvestam, "Study of reaction process on Ni/4H-SiC contact," Materials Science and Technology, vol. 22, no. 10, pp. 1227-1234, 2006.

[17] A. Reina, H. Son, L. Jiao et al., "Transferring and identification of single- and few-layer graphene on arbitrary substrates," Journal of Physical Chemistry C, vol. 112, no. 46, pp. 1774117744, 2008.

[18] L. Zhang, Y. Du, H. Xu, and Z. Pan, "Experimental investigation and thermodynamic description of the Co-Si system," Calphad, vol. 30, no. 4, pp. 470-481, 2006.

[19] A. Das, B. Chakraborty, and A. K. Sood, "Raman spectroscopy of graphene on different substrates and influence of defects," Bulletin of Materials Science, vol. 31, no. 3, pp. 579-584, 2008.

[20] D. S. Lee, C. Riedl, B. Krauss, K. V. Klitzing, U. Starke, and J. H. Smet, "Raman spectra of epitaxial graphene on SiC and of epitaxial graphene transferred to SiO," Nano Letters, vol. 8, no. 12, pp. 4320-4325, 2008.

[21] A. C. Ferrari, J. C. Meyer, V. Scardaci et al., "Raman spectrum of graphene and graphene layers," Physical Review Letters, vol. 97, no. 18, Article ID 187401, 2006.
[22] M. H. Maneshian, M. T. Lin, D. Diercks, and N. D. Shepherd, "Structural and electrical characterization of ohmic contacts to graphitized silicon carbide," Nanotechnology, vol. 20, no. 49, Article ID 495703, 2009.

[23] Z. Zhang, J. Teng, W. X. Yuan, F. F. Zhang, and G. H. Chen, "Kinetic study of interfacial solid state reactions in the $\mathrm{Ni} / 4 \mathrm{H}$ SiC contact," Applied Surface Science, vol. 255, no. 15, pp. 6939-6944, 2009. 

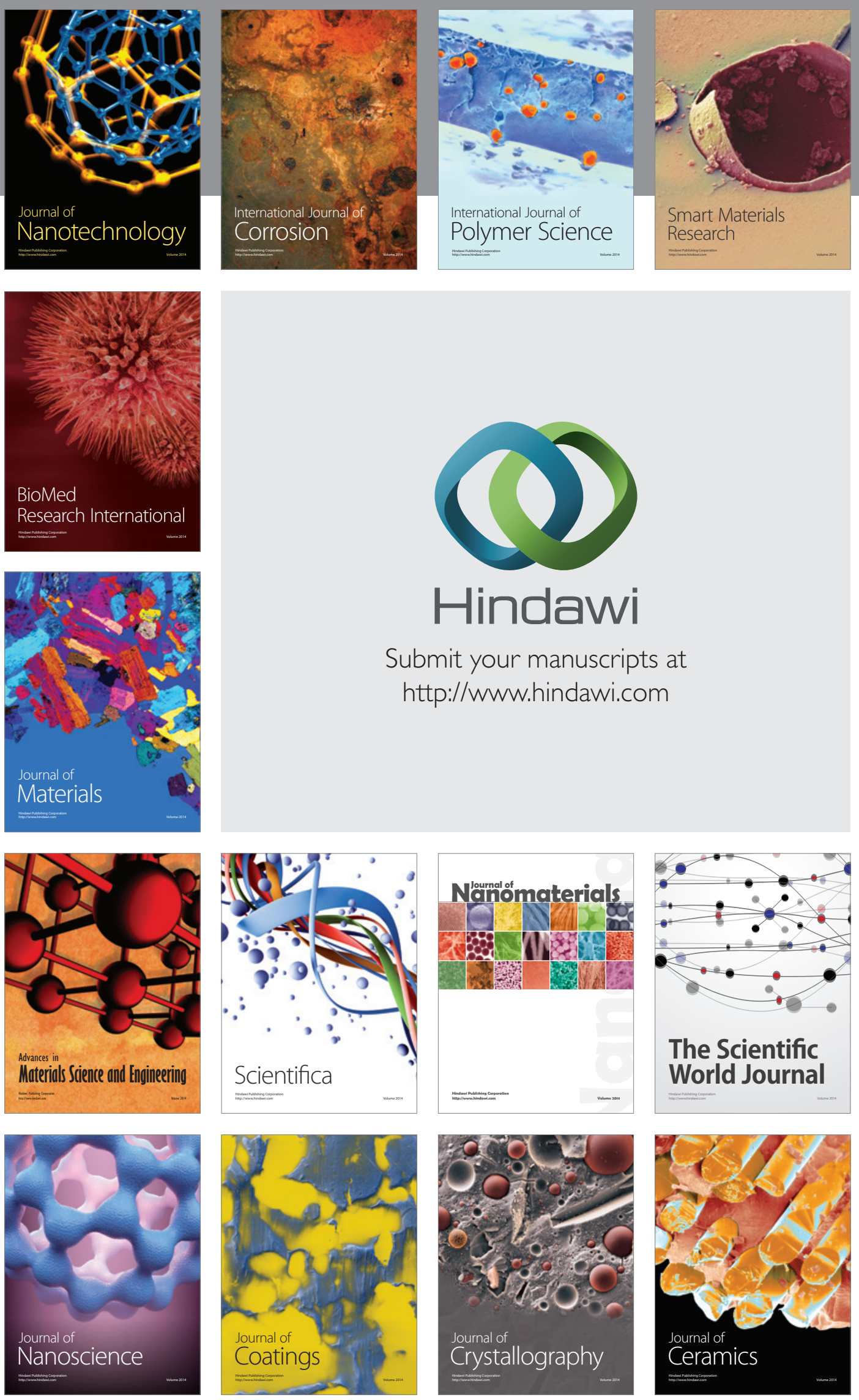

The Scientific World Journal

Submit your manuscripts at

http://www.hindawi.com

\section{World Journal}

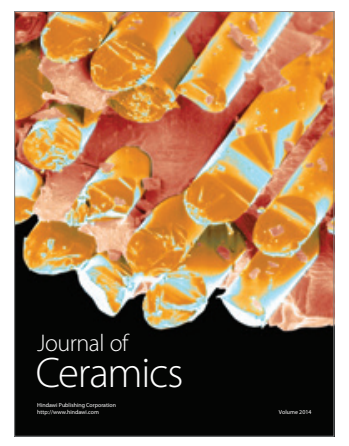

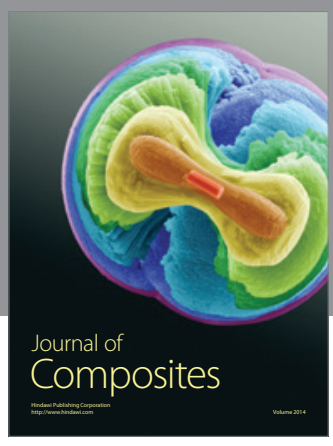
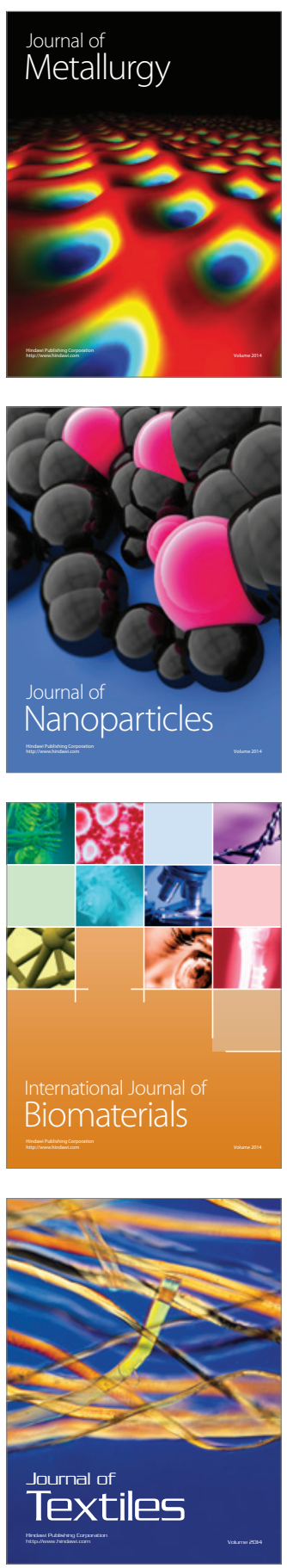